\title{
Enough is not enough: Medical students' knowledge of early warning signs of childhood cancer
}

\author{
J A Geel, ${ }^{1,2}$ MB ChB, FCPaed (SA), Cert Med Onc (Paed), MMed (Paed); B T Stevenson, ${ }^{1}$ MB BCh; R B Jennings, ${ }^{3}$ BA; \\ L E Krook, ${ }^{1}$ BEngSc (BME), MB BCh; S J Winnan, ${ }^{1}$ MB BCh; B T Katz, ${ }^{1}$ MB BCh; T J Fox, ${ }^{1}$ MB BCh; L Nyati, ${ }^{1,4}$ MSc \\ ${ }^{1}$ Department of Paediatrics, Faculty of Health Sciences, University of the Witwatersrand, Johannesburg, South Africa \\ ${ }^{2}$ Division of Paediatric Haematology and Oncology, Department of Paediatrics and Child Health, Charlotte Maxeke Johannesburg \\ Academic Hospital, South Africa \\ ${ }^{3}$ Faculty of Biological Sciences, Wellesley College, Mass., USA \\ ${ }^{4}$ Division of Epidemiology and Biostatistics, School of Public Health, Faculty of Health Sciences, Johannesburg, South Africa
}

Corresponding author: J Geel (jennifer.geel@wits.ac.za)

\begin{abstract}
Background. The reported incidence of childhood cancer in upper-middle-income South Africa (SA) is much lower than in high-income countries, partly due to under-diagnosis and under-reporting. Documented survival rates are disturbingly low, prompting an analysis of potential factors that may be responsible.

Objectives. To determine final-year medical students' level of knowledge of early warning signs of childhood cancer and whether a correlation existed between test scores and participants' age, gender and previous exposure to a person with cancer.

Methods. A two-part questionnaire based on the Saint Siluan mnemonic, testing both recall and recognition of early warning signs of childhood cancer, was administered. The Mann-Whitney-Wilcoxon test was used to assess differences in continuous and count variables between demographic data, experience and responses, and Fisher's exact test and Spearman's rank correlation coefficient were used to determine correlations between demographic data, previous contact with persons with cancer and test scores. A novel equality ratio was calculated to compare the recall and recognition sections and allowed analysis of recall v. recognition.

Results. The 84 participants recalled a median of six signs each (interquartile range 4 - 7) and correctly recognised a median of $70 \%$ in the recognition section, considered a pass mark. There was no correlation between participants' age, gender, previous contact with a person with cancer and recognition scores. Students with previous exposure to a person with cancer had higher scores in the recall section, but this did not achieve statistical significance. Students were able to recognise more signs of haematological malignancies than central nervous system (CNS) malignancies.

Conclusion. The study demonstrated a marked inconsistency between recall and recognition of signs of childhood cancer, with signs of CNS malignancies being least recognised. However, the majority of students could recognise enough early warning signs to meet the university pass standard. Although this study demonstrated acceptable recognition of early warning signs of childhood cancer at one university, we suggest that long-term recall in medical practitioners is poor, as reflected in the low age-standardised ratios of childhood cancer in SA. We recommend increased ongoing exposure to paediatric oncology in medical school and improved awareness programmes to increase early referrals.
\end{abstract}

S Afr Med J 2017;107(7):585-589. DOI:10.7196/SAMJ.2017.v107i7.12211

Childhood cancer constitutes a leading cause of childhood mortality in high-income countries (HICs). ${ }^{[1]}$ Seemingly paradoxically, 5 -year overall survival rates of children with cancer exceed $80 \%{ }^{[2]}$ Unfortunately, despite availability of most of the treatment modalities required to treat childhood cancer, the reported survival rates of South African (SA) children with cancer range between $48 \%{ }^{[3]}$ and $52 \%{ }^{[4]}$ The leading causes of under-5 mortality in SA are intestinal infectious diseases, respiratory and cardiac diseases, disease in the neonatal period, influenza and pneumonia, and other neonatal causes, ${ }^{[5]}$ but as SA's economy has matured from a lower-middleincome country to an upper-middle-income country in the past three decades, ${ }^{[6]}$ it is predicted that diseases more prevalent in HICs will become more important.

The age-standardised ratio of childhood cancer is approximately 130 - 160 per million children in HICs. ${ }^{[2]}$ In SA there are approximately 16.2 million children aged $<15$ years. ${ }^{[7]}$ If the expected incidence rate is applied to the SA population, an annual rate of $900-2500$ new cases of paediatric malignancies would be expected. According to the most recent publication from the South African Paediatric Tumour
Registry ${ }^{[8]} \sim 700$ new cases per year were reported. The reported incidence of childhood cancer is therefore much lower than that in HICs, in part due to under-diagnosis and under-reporting of cases. ${ }^{[9]}$ It has been estimated that two-thirds of SA children with cancer do not reach an appropriate specialist centre, and many of the children who present to a paediatric oncology unit do so with advanced disease. ${ }^{[3]}$ Early warning signs of childhood cancer can easily be mistaken for common and less severe illnesses and can therefore be missed.

The majority of paediatric malignancies are embryonal in origin, as opposed to the epithelial malignancies seen in adults, and potentially have higher survival rates. ${ }^{[2]}$ Paediatric malignancies are usually the result of DNA mutations occurring at an early stage in life, with environmental and lifestyle risk factors playing little or no role. ${ }^{[10]}$ As they have short latency periods and rapid growth ${ }^{[1]}$ more emphasis is placed on early detection, as opposed to the emphasis on preventive measures in adults.

In order to increase the detection and rapid, appropriate referral of children with cancer, a group of doctors working in the paediatric 
oncology field devised and disseminated the Saint Siluan early warning signs of childhood cancer. ${ }^{[3]}$ This mnemonic encompasses signs and symptoms of $>80 \%$ of childhood cancers and has been adopted by the International Society of Paediatric Oncology (SIOP) as an effective tool to increase diagnoses and referrals. ${ }^{[1]}$

It has not yet been established in the available literature whether late referrals of children with cancer due to medical delays (i.e. delays associated with misdiagnoses rather than infrastructural or socioeconomic issues) are associated with poor training or suboptimal recall of the signs of childhood cancer, and there is no documented baseline level of knowledge. While there may be numerous other causes of medical delays, this study focused on medical education alone. It was conducted by medical students as part of a compulsory research block.

\section{Objective}

In order to plan and evaluate awareness programmes, it is essential to establish baseline knowledge in health professionals. The objective of the current study was to determine the level and depth of knowledge of the early warning signs of childhood cancer among final-year medical students at a single university. Given the high number of undiagnosed childhood cancer patients in the SA setting, a secondary aim in selecting this population was to increase awareness about early warning signs of childhood cancer in future medical practitioners.

\section{Methods \\ Population and sample size}

Final-year medical students were targeted in this survey, as various studies have indicated that delays in diagnosis are frequently associated with medical rather than parental delays. ${ }^{[9]}$ The premise was that medical students completing a long period of intensive study and training and about to embark on internship would be expected to have a safe level of knowledge. The study was conducted midway through the year on the 2014 class of final-year medical students. All respondents who gave consent were included. The sample population reflected a convenience sample of the total number of individuals in the population group.

\section{Data collection}

As there are currently no validated questionnaires to determine knowledge of early warning signs of childhood cancer, we used the Saint Siluan mnemonic as a framework to develop a questionnaire, in combination with a questionnaire described in an article from Brazil, ${ }^{[12]}$ a country with economic and social similarities to SA, and used to assess the knowledge of childhood cancer among community health workers. Respondents were also asked to provide demographic data (gender and age), whether they knew anyone (child or adult) with cancer, and whether they had had any exposure to a patient with cancer.

Data were collected by means of self-administration of the questionnaire at a non-compulsory class meeting. It has been reported that questionnaires based on recognition rather than recall yield higher scores, ${ }^{[13]}$ so both assessment modalities were incorporated into the questionnaire in this study. Part 1 comprised an unstructured questionnaire in which participants were asked to recall as many signs of childhood cancer as possible. Part 2 consisted of a structured questionnaire in which participants were asked to indicate which signs from a list of 20 were indeed signs of childhood cancer. This section consisted entirely of actual signs of childhood cancer, i.e. there were no misleading questions, and signs were selected to be representative of the most common paediatric malignancies. The participants were instructed not to turn back to add anything to part 1 once they had turned over to part 2 , to make sure that part 1 accurately tested unprompted recall. There was no time limit, and participants returned the questionnaires when they were completed.

On completion, they were given an awareness leaflet about early warning signs of childhood cancer, provided by the Childhood Cancer Foundation of South Africa (CHOC). The completed questionnaires were then marked by two student members of the research team, and each marked questionnaire was checked by a paediatric oncologist (JG).

\section{Statistical analysis}

In the recall section, participants were required to furnish as many signs of childhood cancer as they could remember. There was therefore no finite score to attain (i.e. no denominator with which to compare the recall answers of the study population). An equality ratio was thus created to assess the consistency between the recall and recognition abilities of the students. The ratio was calculated by the following formula: 'number of correct recall answers/number of correct recognition answers' for each student. Participants who did not provide a single correct answer in recall and/or recognition were excluded from the equality ratio (five participants). A score of 1 in the equality ratio was considered ideal, as this hypothetically shows an equivalence between ability to recall a fact unprompted and recognising the same fact when prompted. Continuous and count data (age, recall, recognition and equality ratio) were assessed for normality and presented as medians with interquartile ranges (IQRs). The Mann-Whitney-Wilcoxon test was used to assess differences in continuous and count variables between demographic data, experience and responses. Spearman's rank correlation was used to assess associations between continuous and count variables. All analyses were performed at the $95 \%$ confidence interval using Stata Statistical Software, version 13 (StataCorp, USA). Statistical significance was defined as $p<0.05$.

\section{Ethics and institutional approval}

Permission to conduct the study was obtained from the Dean of the Faculty of Health Sciences and the Human Research Ethics Committee (HREC), University of the Witwatersrand (Wits) (ref. no. M140348).

\section{Results}

The questionnaires were completed by 84 students at the noncompulsory class meeting, at which 130 of the 220 students registered were present, representing a response rate of $84 / 130$ (64.6\%). Respondents included 62 females, 21 males and one of unspecified gender, with a median age of 24 years (range $22-35$ ). Of the students who completed the questionnaire, $51(60.7 \%)$ reported having been involved in the management of a person with cancer, 50 (59.5\%) having both known and been involved in the medical management of a person with cancer, and one having neither known nor been involved in the management of a person with cancer. No student reported having only known a person with cancer.

The median number of correctly recalled responses was 6 (IQR 4 $7)$. Neither gender nor age was associated with higher scores in the recall section ( $p=0.390$ and $p=0.870$, respectively). In addition, no correlation was detected between higher recall scores and knowing a person with cancer $(p=0.725)$ or having been involved in the management a patient with cancer $(p=0.807)$.

The participants correctly identified $70 \%$ of the signs from the list of 20 options; $70.2 \%$ of participants could recognise at least 
$60 \%$ (considered a pass at Wits Medical School) of the listed signs, while $44.1 \%$ of participants could correctly identify more than $75 \%$ (considered a first-class pass) of the signs (range 0 - 100\%) (Fig. 1). No correlation was found between scores achieved and the participant's gender $(p=0.650)$ or age $(p=0.850)$ in the recognition section.

The participants had high scores when asked to identify signs relating to haematological malignancies such as unexplained bleeding, correctly identified by $85.7 \%$, easy bruising $(86.9 \%)$ and fatigue (85.7\%) (Fig. 2). Symptoms such as unexplained fever (81.0\%) and loss of weight and appetite (88.9\%) were also well recognised. Participants showed poor recognition of neurological signs such as a change in balance $(44.1 \%)$ and an enlarging head (41.7\%), as well as one of the signs of leukaemia, aching joints (48.8\%).

The relationship between the recall and recognition sections was tested using Spearman's rank correlation coefficient, and these were determined to be independent of each another (Spearman's $\rho=-0.033$, $p=0.763$ ). The median equality ratio score for the participants was 0.47 (IQR $0.29-0.58$ ), indicating that the majority of the students scored higher in the recognition section than in the recall section.

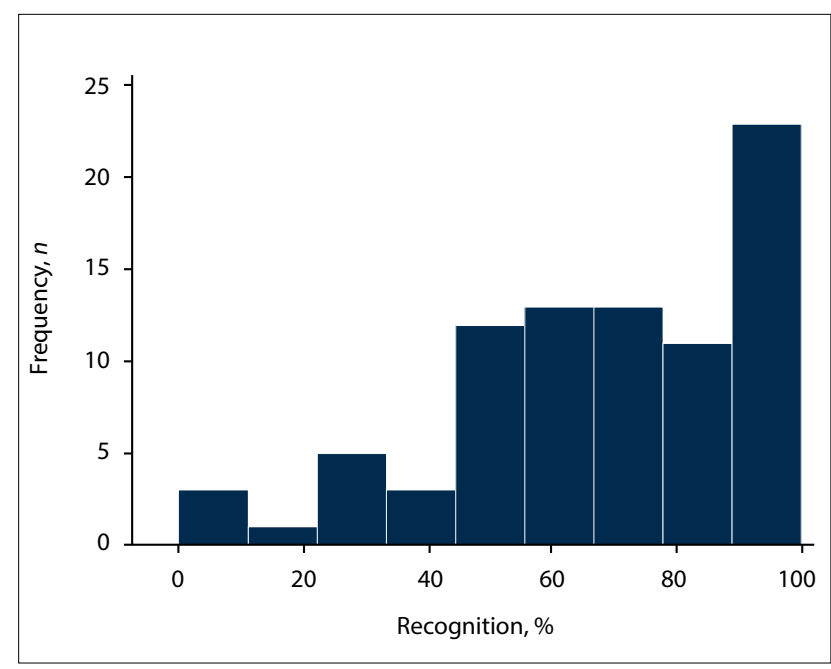

Fig. 1. Histogram of participant scores achieved in the recognition section of the test.

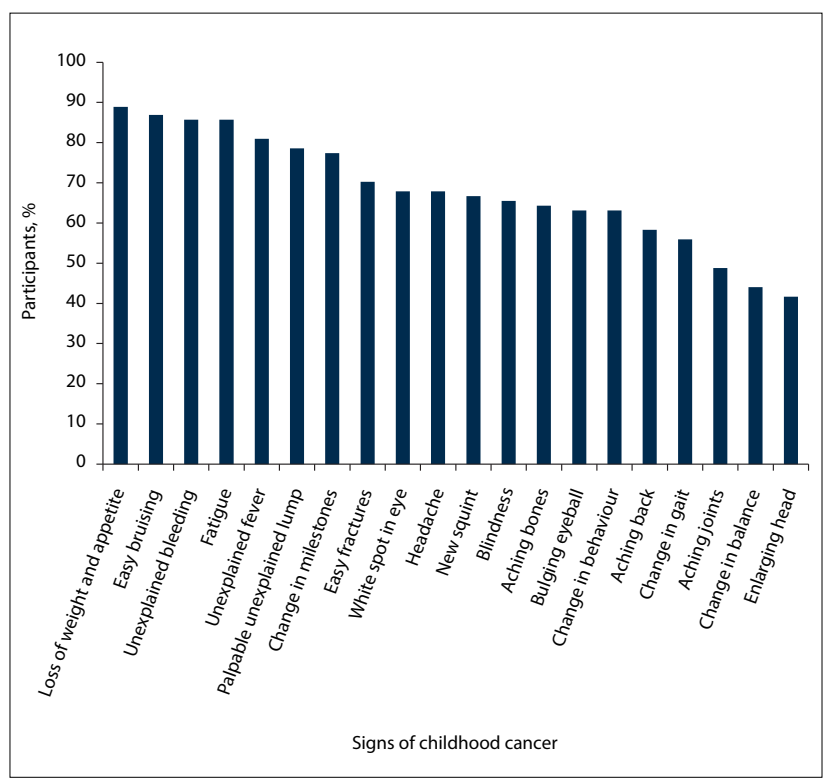

Fig. 2. Percentage of student participants who correctly identified the early warning signs of childhood cancer.
There were negative correlations between test scores and gender, personal experience, clinical experience, and the combination of clinical and personal experience (Fig. 3).

\section{Discussion}

This student-initiated study aimed to determine knowledge of early warning signs of childhood cancer in final-year medical students at a single university. Both recall and recognition questions were used to assess knowledge of the early warning signs. As predicted, students attained higher scores in the recognition section than in the recall section. Recognition questions prompt students' memories, allowing them to recall signs that they may otherwise not have remembered as signs of childhood cancer. ${ }^{[13]}$

Previous clinical experience of a patient with cancer was found to have a statistically significant impact on the equality ratio in the study population, suggesting that clinical experience is associated with an increased equality between recall and recognition of childhood cancer symptoms. The overall median of 0.47 suggests a discrepancy in the recall v. recognition abilities of the students. With the population mean being $<1$, and given that the mean score in the recognition section was $68.1 \%$, it may be inferred that medical students are trained to recognise signs and symptoms rather than being able to recall them from memory.

Signs indicating central nervous system (CNS) malignancies were under-recognised in this study, fewer than half the respondents being able to recognise neurological deficits and an enlarging head as potential signs of childhood cancer (Fig. 2). Certain presenting symptoms are related to diagnostic delays, with CNS tumours having both the longest median delay and the highest risks for physician delay. ${ }^{[1,9,14]}$ As CNS malignancies are the second most common form of childhood cancer in SA, ${ }^{[8]}$ the observation that so many of our students were unaware of their signs is particularly concerning. Longer diagnostic delays in CNS malignancies increase the likelihood that children will develop cognitive and learning difficulties as well as irreversible neurological deficits. ${ }^{[14]}$ In contrast, children who are diagnosed earlier in the disease process tend to have fewer long-term complications. ${ }^{[14]}$

State primary- and secondary-level healthcare facilities may be where the child with cancer first encounters healthcare professionals, including junior doctors, who could detect early warning signs. Without adequate familiarity with these signs, delays in diagnosis are almost inevitable. ${ }^{[12]}$ Early diagnosis enables timely treatment and may prevent unnecessary complications. ${ }^{[14]}$ The importance of educating the healthcare community on early warning signs has been documented over more than two decades. ${ }^{[15]}$ It has been agreed that a wider range of medical professionals should be educated, and that knowledge of early warning signs of childhood cancer should not be limited to paediatric oncologists. ${ }^{[9,16]}$ In SA there is a clear need to increase awareness, given that physician delays tend to be longer than patient delays, and that $>50 \%$ of patients are initially misdiagnosed. ${ }^{[9]}$

A significantly longer overall delay, including physician delay, has been documented when a child's presenting symptom is pain, ${ }^{[12]}$ and the current study confirmed that aching joints and bones are poorly recognised, suggesting the possibility that these will be missed in a clinical setting. Poor knowledge of neurological signs should be addressed by placing more emphasis on neurological tumours of childhood, with increased patient exposure in the academic hospitals during undergraduate training.

\section{Study limitations}

The survey method, while approximating an undergraduate testing experience and approved by the HREC, was not formally approved 


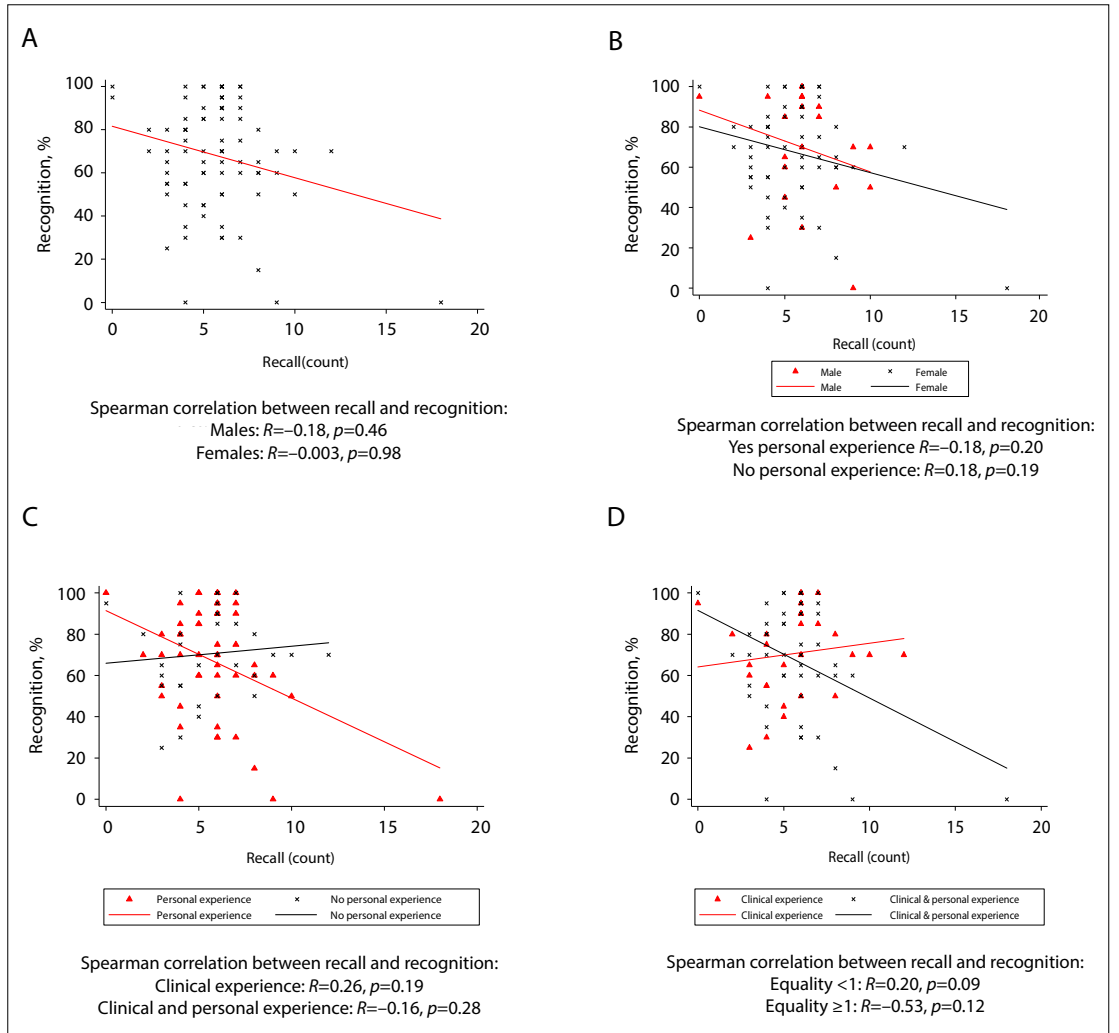

Fig. 3. Negative correlations between gender (A), personal experience (B), clinical experience (C) and the combination of clinical and personal experience $(D)$ and test scores.

as a testing method by an examinations committee and therefore cannot be directly compared with university test scores. The questionnaire was completed in an uncontrolled environment, and not all students were in attendance. Consequently, the study participants could have belonged to a specific subset in the class comprising individuals who were more likely to attend formal academic training sessions and who therefore might be expected to score higher in the questionnaire, and not be a true representation of the class in its entirety. The response rate of $64.6 \%$ is considered adequate, but the possibility that the scores were influenced by selection bias cannot be ignored, and the sample size was not as large as was anticipated. The age range of the class was narrow, as was expected, and it was therefore not possible to detect any difference in scores associated with age. The wording of the question: 'Do you know anyone (child or adult) who has/had cancer', allowed for ambiguity in its interpretation. The aim was to determine whether the participants had personal knowledge of someone with cancer, but the question allowed for overlap between those who knew someone personally and those who had had clinical contact with a patient with cancer, potentially allowing both groups to answer 'yes'. The equality ratio devised for this study is a theoretical concept and has not yet been validated. The survey was conducted in a single SA university and the findings may therefore not be applicable to other settings.

\section{Recommendations}

Healthcare workers should be skilled in recognising early warning signs of childhood cancer in order to diagnose and refer patients appropriately and promptly. All healthcare workers who interact with children should be made aware of these warning signs to decrease delays. These professionals include but are not limited to primary healthcare workers, general practitioners, paediatricians, and traditional and complementary practitioners. There may be a need for reassessment of the methods of medical student training regarding childhood cancer. Of necessity, as a result of the growing numbers of students in medical schools nationwide, examinations using the multiple-choice format have assumed greater importance. We postulate that this may have contributed to the skewing of learning towards recognition and away from recall. While it would take studies with far greater scope than this one to detect or confirm an association between learning and teaching methods and early detection rates of childhood cancers, it is recommended that attention be directed to this issue. The unacceptably low survival rate of children with cancer in SA should be addressed as a matter of urgency. This study contributes to the small but growing body of data addressing this issue, and should be taken into account when planning awareness campaigns.

\section{Conclusion}

The low survival rate of children with cancer in SA requires urgent attention and research to determine its many causes. While medical education is not the only factor impacting on this issue, it is an important one and can be addressed in the formal university setting.

No correlation was demonstrated between the ability of students in this study to correctly recall answers and knowledge of a person with cancer. Moreover, no association was observed between their ability to recognise signs of cancer and previous clinical experience. Although a score at the level of a university pass was attained by $70 \%$ of final-year medical students when asked to recognise early signs of childhood cancer, these students demonstrated a lack of consistency between recall and recognition, with overall reliance on recognition based on analysis of the equality ratio. This novel tool shows potential for the ongoing evaluation of levels of knowledge of such concepts. Participants demonstrated higher levels of awareness of signs relating to haematological cancers than signs of CNS malignancies.

Improving survival rates of children with cancer will require a thoughtful, multipronged approach, and increasing awareness at medical school represents the foundation of this undertaking. Despite our respondents achieving the required scores to pass university examinations, we contend that more effort may be required to increase the depth and recall of knowledge. Emphasis should be placed on increasing contact time with patients. Enough is not enough ...

Acknowledgements. The authors wish to thank Drs S Carrim, Z Dlamini, K Gama, L Matlala, E Milwid, K Naicker, J Omony, N Rasool and $\mathrm{C}$ von Bardeleben for their input into the original research project, Ms Adri Ludick of CHOC for information leaflets and ongoing assistance, and Prof. Ben Sartorius for statistical assistance. Author contributions. JAG conceptualised the study, supervised the project and wrote the article. BTS, LEK, SW, BTK and TJF collected and analysed data, wrote the initial report and contributed to the article. RBJ contributed to statistical analysis and co-wrote the manuscript. LN performed statistical analysis and contributed to the article.

Funding. None.

Conflicts of interest. None. 
1. Dang-Tan T, Franco EL. Diagnosis delays in childhood cancer. Cancer 2007;110(4):703-713. http:// dx.doi.org/10.1002/cncr.22849

2. Kaatsch P. Epidemiology of childhood cancer. Cancer Treat Rev 2010;36(4):277-285. http://dx.doi. org/10.1016/i.ctrv.2010.02.003

3. Poyiadjis S, Wainwright L, Naidu G, et al. The Saint Siluan warning signs of cancer in children:

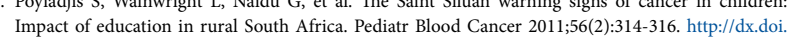
Impact of education in $r$
org $/ 10.1002 / \mathrm{pbc} .22853$

4. Stones DK, de Bruin GP, Esterhuizen TM, et al. Childhood cancer survival rates in two South African units. S Afr Med J 2014;105(7):501-504. http://dx.doi.org/10.7196/SAMJ.7882

5. Nannan N, Dorrington RE, Laubscher R, et al. Under-5 Mortality Statistics in South Africa: Shedding Some Light on the Trends and Causes 1997 - 2007. Cape Town: South African Medical Research Council, 2012. http://www.mrc.ac.za/bod/MortalityStatisticsSA.pdf (accessed 1 June 2017).

6. World Bank. Data for upper middle income, South Africa. http://data.worldbank.org/?locations=XTZA (accessed 16 November 2016)

7. Statistics South Africa. Media release, mid-year population estimates 2014. http://www.statssa.gov. $\mathrm{za} /$ ? $=2990$ (accessed 18 June 2015).

8. Stefan DC, Stones DK. The South African Paediatric Tumour Registry - 25 years of activity. S Afr Med J 2012;102(7):605-606. DOI:10.7196/SAMJ.6113

9. Stefan DC, Siemonsma F Delay and causes of delay in the diagnosis of childhood cancer in Africa. Paediatr Blood Cancer 2010;56(1):80-85. http://dx.doi.org/10.1002/pbc.22714

10. Pizzo PA, Poplack DG. Principles and Practice of Pediatric Oncology. 4th ed. Philadelphia: Lippincott Williams \& Wilkins, 2012 .
11. Perilongo G, Craft A, Jereb B, Wagner H, D'Angio GJ, for the SIOP History Writing Group. The SIOP story: An informal history of the International Society of Pediatric Oncology. Pediatr Blood Cancer story: An informal history of the Internationa
2016;63(S4). http://dx.doi/10.1002/pbc.26170

12. Workman GM, Ribeiro RC, Rai SN, Pedrosa A. Pediatric cancer knowledge: Assessment of knowledge of warning signs and symptoms for pediatric cancer among Brazilian community health workers. J Cancer Educ 2007;22(3):181-185. http://dx.doi.org/10.1007/BF03174334

13. Stubbings S, Robb K, Waller J, et al. Development of a measurement tool to assess public awareness of cancer. Br J Cancer 2009;101(Suppl 2):S13-S17. http://dx.doi/10.1038/sj.bjc.6605385

14. Raney RB, O’Donnell JF, Brooks CM, et al. Pediatric oncologists' assessment of oncology educatio in US medical schools: Cancer Education Survey II. J Cancer Educ 1994;9(3):141-144. http://dx.d oi. 10.1080/08858199409528295

5. Chantada G, Fandiño A, Manzitti J, et al. Late diagnosis of retinoblastoma in a developing country. Arch Dis Child 1999;80(2):171-174. http://dx.doi.org/10.1136/adc.80.2.171

16. Wilne S, Koller K, Collier J, et al. The diagnosis of brain tumours in children: A guideline to assis healthcare professionals in the assessment of children who may have a brain tumour. Arch Dis Child 2010;95(7):534-539. http://dx.doi.org/10.1136/adc.2009.162057

Accepted 27 February 2017 